\title{
Aproveitamento Teórico-Prático da Disciplina Anatomia Humana do Curso de Fisioterapia
}

\author{
Students' Theoretical-Practical Harnessing of \\ the Subject of Human Anatomy in \\ Physiotherapy Courses
}

Rodrigo Moreira Arruda ${ }^{I}$

Cintia Regina Andrade Sousal

PALAVRAS-CHAVE

- Ensino;

- Aprendizagem;

- Anatomia;

- Educação Médica.

\section{KEYWORDS}

- Teaching;

- Learning;

- Anatomy;

- Medical Education.

Recebido em: 21/08/2013

Aprovado em: 10/09/2013

\section{RESUMO}

A Anatomia Humana estuda as estruturas do corpo humano e as relações entre elas. Assim, seu ensino a estudantes da área da saúde, incluindo alunos de Fisioterapia, é de extrema importância. É necessário um bom aproveitamento nesta disciplina para se tornar um bom profissional, porém as estratégias de ensino-aprendizagem nesta disciplina têm sido bastante discutidas. Com base nesta premissa, este estudo teve por objetivos avaliar o aproveitamento teórico-prático dos discentes do curso de Fisioterapia na disciplina Anatomia Humana, bem como descrever as facilidades e dificuldades enfrentadas e suas implicações durante o curso. Para tanto, foi elaborado um questionário específico e utilizada também a avaliação dos professores realizada pelo Colegiado de Fisioterapia. O tratamento estatístico foi composto por análise descritiva e correlação de Pearson, sendo os dados analisados pelos programas Microsoft Excel® 2007 e Graphpad Prism ${ }^{\circledR}$. Não se observou correlação entre desempenho do aluno e grau de satisfação diante de diferentes parâmetros analisados neste trabalho. O estudo revelou que os alunos apresentam aproveitamento satisfatório, mas há grandes insatisfações quanto à forma como a disciplina é conduzida.

\section{ABSTRACT}

Human Anatomy is the study of the structures of the human body and the relationship between these structures. Its teaching to students in the healthcare field, including physiotherapy, is thus of utmost importance. A sound basis in the subject is necessary to becoming a sound professional, however strategies for the teaching of this subject and its learning have been the subject of much debate. Based on this prerogative, this study aimed to evaluate the subject's theoretical and practical use by physiotherapy students on the discipline's Human Anatomy course, as well as to describe the ease and difficulties experienced and their implications during the course. We therefore devised a questionnaire and used teacher assessment conducted by the College of Physiotherapy. Statistical analysis consisted of a descriptive analysis and Pearson correlation, and data was analyzed by Microsoft Excel $® 2007$ and GraphPad Prism ${ }^{\circledR}$. There was no correlation between student performance and satisfaction in terms of the different parameters analyzed in this work. The study revealed that students make satisfactory use of the subject, but that there is great dissatisfaction concerning the way the course is conducted. 


\section{INTRODUÇÃO}

O estudo da Anatomia sempre exerceu fascínio sobre o homem através dos tempos. Esta ciência, que estuda macro e microscopicamente a constituição e o desenvolvimento dos seres organizados, dentre eles o homem, é sujeito de pesquisa da disciplina Anatomia Humana ${ }^{1}$.

A trajetória desta área de conhecimento, desde a pré-história até os tempos atuais, fortalece a importância destes conhecimentos, garantindo o consenso de que esta é uma das disciplinas básicas para a formação de toda profissão da área da saúde ${ }^{2}$. Dentre essas profissões encontra-se a do fisioterapeuta. Sem as noções desta matéria, tanto o profissional quanto o estudante ficam inaptos para examinar, diagnosticar e tratar seu paciente ${ }^{3}$.

Dentre as diversas competências adquiridas por meio do estudo da Anatomia destacam-se: demonstrar habilidades na identificação e dissecação de peças anatômicas de segmentos corporais; compreender as funções de órgãos e sistemas e correlacionar ossos, articulações, músculos, vasos e nervos com as regiões topográficas ${ }^{4}$. Para alcançar esse conhecimento, são necessários conceitos teóricos (desenvolvidos na sala de aula), que, aplicados à prática em laboratório, demandam do professor encarar desafios em busca de novas metodologias para que a Anatomia seja mais bem assimilada pelos alunos ${ }^{5}$.

Neste contexto é que se insere a importância do processo de ensino-aprendizagem em Anatomia, transformando a prática de uma aprendizagem de certa forma mecânica dos conhecimentos anatômicos em uma aprendizagem mais dinamizada ${ }^{6}$.

Seguindo a compreensão de que a Anatomia Humana desempenha um grande papel na formação dos discentes em Fisioterapia, este estudo tem por objetivos avaliar o aproveitamento teórico-prático dos discentes do curso de Fisioterapia da Universidade do Sudoeste da Bahia na disciplina Anatomia Humana, bem como descrever as facilidades e dificuldades encontradas durante o andamento dessa disciplina, como também suas implicações durante o curso.

\section{METODOLOGIA}

Neste estudo optou-se por desenvolver uma pesquisa com abordagem descritiva, exploratória, com metodologia quanti-qualitativa, de acordo com os objetivos previamente citados da pesquisa realizada na Universidade Estadual do Sudoeste da Bahia (Uesb), no curso de bacharelado em Fisioterapia.

A população pesquisada foi composta por 257 discentes matriculados regularmente no curso de bacharelado em Fisioterapia da Uesb, sendo que a amostra inicial foi de 167 discentes selecionados pelo método probabilístico, do tipo aleatório simples. Segundo os critérios de inclusão, os discentes do curso de Fisioterapia da Uesb deveriam se encontrar do quarto semestre letivo em diante e já haver cursado as disciplinas de Anatomia Humana ministradas nos três primeiros semestres do curso, sendo elas: Anatomia I (anatomia básica dos sistemas do corpo humano); Anatomia II (anatomia do sistema musculoesquelético); Anatomia III (neuroanatomia). Os discentes que ainda estivessem cursando as disciplinas Anatomia Humana não seriam considerados, segundo o critério de exclusão. Devido a alguns fatores que inviabilizaram a aplicação do questionário, a amostra foi de 120 discentes.

Os dados foram coletados por meio de um questionário com perguntas objetivas (metodologia quantitativa) e questões subjetivas (metodologia qualitativa). O questionário utilizado nesta pesquisa foi adequado pelos autores do estudo para atender a seus objetivos e continha 22 perguntas, estruturadas em quatro partes: identificação (6 perguntas), avaliação do discente (10 perguntas), avaliação da disciplina Anatomia Humana (5 perguntas) e avaliação do docente (1 pergunta). Foi usado também um questionário semiestruturado de avaliação docente, aplicado aos discentes pelo Colegiado do curso de Fisioterapia, nas turmas do período de 2009.1 até 2011.1 para Anatomia I, de 2009.1 até 2011.2 para Anatomia II e de 2009.1 até 2012.1 para Anatomia III.

Este conjunto de dados foi analisado sob a ótica da estatística descritiva e da análise de correlação de Pearson, utilizando os programas Microsoft Excel® 2007 e Graphpad Prism ${ }^{\circledR}$. Este estudo foi aprovado pelo Comitê de Ética em Pesquisa da Universidade Estadual do Sudoeste da Bahia (CEP/Uesb), CAAE 10688512.7.0000.0055, de forma a respeitar as diretrizes da Resolução 196/966 do Conselho Nacional de Saúde sobre a participação de seres humanos em pesquisas.

\section{RESULTADOS E DISCUSSÃO}

O curso de bacharelado em Fisioterapia da Universidade Estadual do Sudoeste da Bahia conta com a disciplina Anatomia Humana desde o início de sua implementação no campus de Jequié (BA), sendo dividida em três disciplinas: Anatomia I, Anatomia II e Anatomia III, dispostas nos três primeiros semestres deste curso, respectivamente. Cento e vinte discentes, regularmente matriculados, responderam ao questionário desta pesquisa, dividido em quatro partes: identificação; avaliação do discente; avaliação da disciplina Anatomia Humana; avaliação do docente.

Na identificação do discente, foi observado um perfil que corresponde ao predomínio de indivíduos do sexo feminino (73,3\%), sendo que a maioria tinha 22 anos (20,8\%), com média de 21,7 anos, procedentes da cidade de Jequié $(29,1 \%)$, não cotistas $(73,3 \%)$, tendo $52,5 \%$ do universo amostral cursado o en- 
sino fundamental ou médio em escola pública. Estes dados foram corroborados por outro estudo com discentes de cursos de saúde matriculados na disciplina Anatomia, que demonstrou predomínio do sexo feminino e média de idade de 20 anos, indicando, nesta perspectiva, um índice maior de mulheres no nível superior na área da saúde e de indivíduos jovens ${ }^{7}$.

Na segunda parte do questionário, referente à avaliação do discente, pôde-se observar que 47,5\% (Anatomia I) e 45,8\% (Anatomias II e III) dos discentes relataram ter afinidade média com a disciplina Anatomia Humana. A média dos valores relacionados ao grupo que relatou ter alta afinidade foi de $28,88 \%$, enquanto o grupo com pouca afinidade foi de $23,33 \%$. Os dados sugerem grande aceitação da atividade de monitoria, tendo sido observado que $97,5 \%$ dos alunos julgaram importante a presença do monitor durante o andamento das disciplinas Anatomia I, II e III. Nesta perspectiva, a presença de monitores em conjunto com assistentes de laboratório e espaço físico próprio para aplicação da disciplina são elementos-chave para o sucesso de uma boa aprendizagem em Anatomia ${ }^{8,9}$.

Ao serem perguntados se a nota final foi compatível com o conhecimento adquirido, 42,5\% dos discentes assinalaram que nas Anatomias I e II foi compatível, enquanto na Anatomia III este índice foi de $61,6 \%$, sugerindo um grau de satisfação maior dos alunos na Anatomia III. Cerca de 43,3\% dos alunos de Anatomia I e 31,67\% de Anatomia III revelaram ter pouca dificuldade de aprendizagem, contrastando com os valores observados em Anatomia II, que foi de 9,2\%. Maior rendimento foi observado em Anatomia III, onde 61,67\% dos alunos revelaram ter notas acima de 8 , contrastando com as Anatomias II (20\%) e Anatomia I (17,5\%), para este mesmo nível de rendimento.

Ao se analisar o índice de aprovação, tem-se que 81,67\% dos alunos nunca foram reprovados nas disciplinas de Anatomia (I, II e III). Observa-se um índice de aprovação final de $61,67 \%$ para Anatomia I, 42,5\% para Anatomia II e 80,83\% para Anatomia III. Dos 18,3\% que foram reprovados, a Anatomia II apresenta maior frequência de reprovação, com $76 \%$ deste universo amostral. Estas informações foram apoiadas por outro estudo ${ }^{10}$, que, ao analisar a taxa de reprovação da Anatomia nos cursos de saúde, dentre eles um curso de Fisioterapia, verificou um índice maior de reprovação em Anatomia II, que variou de $31,25 \%$ a $53,70 \%$. Tais resultados foram justificados pelo fato de na Anatomia I os assuntos abordados terem semelhança com assuntos prévios do ensino médio, ao passo que na Anatomia II são novos assuntos e em quantidade maior.

Com relação às perguntas qualitativas do questionário, na primeira pergunta sobre as dificuldades durante a disciplina, podem-se enquadrar as respostas em cinco categorias: (1) quantidade de conteúdo e material prático $(30,0 \%)$, destacando-se: "Quantidade de conteúdos trabalhados em curto espaço de tempo" e "Dificuldades nas aulas práticas devido à falta de peças anatômicas e ao estado de conservação das existentes"; (2) memorização e localização das estruturas (30,8\%), podendo ser destacado: "O estudo da Anatomia envolve o conhecimento de muitos detalhes e abordagem de um assunto descritivo, o que faz com que seja uma disciplina decoreba"; (3) metodologia e didática do professor (34,2\%), com menções como: “Dificuldades em relação à metodologia aplicada, que nos forçava a decorar os assuntos e as disciplinas, principalmente a Anatomia II, em que não havia aplicação clínica"; (4) relação prática x teoria para o curso de Fisioterapia $(16,7 \%)$, ressaltando-se: "Pouca ou nenhuma correlação clínica, dificultando o entendimento de outras disciplinas; o estudo da Anatomia é estático; se fosse estudada com outras disciplinas, como Fisiologia e Cinesiologia, seria mais fácil fazer associações"; (5) nenhuma dificuldade $(4,2 \%)$, enfatizada por um discente "Nenhuma, motivada pelo interesse e afinidade com a disciplina".

Tais dificuldades são evidenciadas por vários autores, os quais inferem que o fato de o ensino em Anatomia ter sido vinculado ao ciclo básico dos cursos de saúde nas universidades gera problemas, principalmente pela necessidade de fazer uma abordagem integrada com outras disciplinas; além disso, a proposta pedagógica em uso não ensina a vincular a teoria com a prática, para que o aluno saiba reter o conhecimento e o transpor para a comunidade $\mathrm{e}^{11,12,13}$. Neste sentido, tem sido reforçada a ideia de que a disciplina de Anatomia Humana tem um caráter de memorização, o que traz grandes dificuldades para os discentes, que, ao invés de aprenderem a Anatomia, se preocupam em memorizá-la ${ }^{14}$.

Quando perguntados sobre a importância da disciplina no estágio, houve unanimidade quanto a sua fundamental importância, destacando-se a resposta: "Essencial, é impossível diagnosticar, avaliar e tratar sem conhecer anatomia e suas correlações. Anatomia é uma das bases do trabalho de um fisioterapeuta". Todavia, os estudantes de saúde somente percebem a importância da Anatomia quando se encontram frente ao paciente, uma situação tão corriqueira na vida do profissional da área de saúde, momento no qual ele tem a oportunidade de comprovar todo o conhecimento adquirido ${ }^{15}$.

Quando indagados sobre a importância dos conhecimentos da Anatomia para as outras disciplinas, eles citaram 29 disciplinas; dentre elas, Cinesiopatologia foi mencionada por $55,0 \%$ e Cinesiologia por 51,7\% dos entrevistados. Tais dados são reforçados no estudo sobre a importância da Anatomia para o estudante da área da saúde, demonstrando que a Anatomia Humana, na opinião dessa população, influencia muito o de- 
sempenho de outras disciplinas ${ }^{16}$. No entanto, o método de ensino da Anatomia Humana vai de encontro a uma metodologia que empregue correlações com outras disciplinas, não favorecendo um currículo integrado, no qual o estudante seria capaz de abordar uma problemática de forma mais ampla e melhor . $^{8}$

Na terceira parte do questionário, referente à avaliação da disciplina Anatomia Humana, a maioria dos alunos entrevistados concorda com a existência dos pré-requisitos entre as Anatomias (72,88\%) e com a alocação das disciplinas em seus respectivos semestres (79,38\%). Quanto aos métodos de avaliação, a prova escrita foi empregada na avaliação do rendimento de todos os entrevistados. $\mathrm{O}$ estudo dirigido e a prova prática perfizeram $40,80 \%$ e $34,10 \%$ dos entrevistados, respectivamente. Poucos alunos foram avaliados de modo processual $(17,60 \%)$ ou por avaliação oral $(9,10 \%)$ (Figura 1$)$.

\section{FigurA 1}

Processo avaliativo submetido aos discentes do curso de Fisioterapia

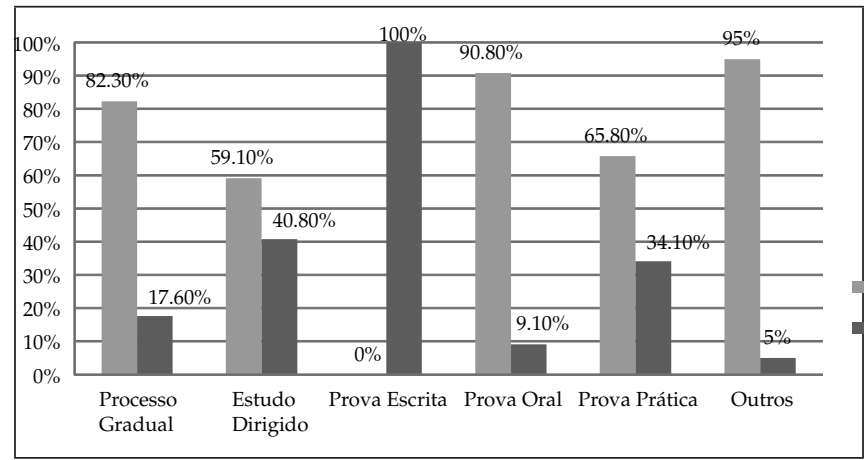

Na avaliação da satisfação dos alunos de Fisioterapia, a Tabela 1 indica alguns aspectos da disciplina Anatomia Humana. De modo geral, os dados sugerem uma superioridade na taxa média de alunos insatisfeitos $(56,67 \%)$ ao se analisarem conjuntamente as três Anatomias. Foram observadas taxas de insatisfação de 58,06\% para Anatomia II e de 66,94\% para Anatomia III. No entanto, os dados sugerem menor taxa de insatisfação para Anatomia I, correspondendo a 45\% (55\% de satisfeitos) dos alunos entrevistados. Neste conjunto de dados, evidenciamos os valores de $86,7 \%$ de satisfação quanto ao conteúdo de Anatomia I e de 79,2\% de insatisfação quanto ao uso de peças anatômicas em Anatomia III. Tal insatisfação também está presente no estudo de Costa et al. ${ }^{7}$, o qual afirma que o uso de tais peças visa apenas mostrar a localização das estruturas, o que muitas vezes não é possível devido ao número de peças e a sua qualidade.

Com relação às perguntas qualitativas do questionário (avaliação da disciplina Anatomia Humana), as sugestões e contribuições para a disciplina podem ser categorizadas em quatro grupos: (1) mudanças na metodologia e didática dos professores $(43,3 \%)$, evidenciando: “Uma didática que fosse mais bem aproveitada pelos alunos, que aprendessem realmente a Anatomia e não decorassem seu conteúdo para serem aprovados na matéria"; (2) aumento de carga horária, viabilizando a execução da anatomia palpatória (23,3\%), destacando: "Anatomia palpatória deveria ser um dos conteúdos-chave da disciplina"; (3) melhores recursos práticos (33,3\%), ressaltando: "Maior número de peças anatômicas e em bom estado de conservação"; (4) correlacionar o conteúdo com a Fisioterapia (20,8\%), destacando: “Abordagem mais específica da área de

TABela 1

Satisfação dos discentes do curso de bacharelado em Fisioterapia quanto à avaliação da disciplina Anatomia Humana

\begin{tabular}{|c|c|c|c|c|c|c|}
\hline & \multicolumn{2}{|c|}{ Anatomia I } & \multicolumn{2}{|c|}{ Anatomia II } & \multicolumn{2}{|c|}{ Anatomia III } \\
\hline & Insatisfeito & Satisfeito & Insatisfeito & Satisfeito & Insatisfeito & Satisfeito \\
\hline Conteúdo & $13,3 \%$ & $86,7 \%$ & $34,2 \%$ & $65,8 \%$ & $41,7 \%$ & $58,3 \%$ \\
\hline Carga Horária & $15,8 \%$ & $84,2 \%$ & $39,2 \%$ & $60,8 \%$ & $45,8 \%$ & $54,2 \%$ \\
\hline Metodologia de ensino & $50,8 \%$ & $49,2 \%$ & $68,3 \%$ & $31,7 \%$ & $72,5 \%$ & $27,5 \%$ \\
\hline Apoio aos alunos & $59,2 \%$ & $40,8 \%$ & $63,3 \%$ & $36,7 \%$ & $72,5 \%$ & $27,5 \%$ \\
\hline $\begin{array}{l}\text { Estrutura das aulas } \\
\text { práticas }\end{array}$ & $52,5 \%$ & $47,5 \%$ & $64,2 \%$ & $35,8 \%$ & $78,3 \%$ & $21,7 \%$ \\
\hline Método de avaliação & $53,3 \%$ & $46,7 \%$ & $71,7 \%$ & $28,3 \%$ & $66,7 \%$ & $33,3 \%$ \\
\hline Uso de peças anatômicas & $46,7 \%$ & $53,3 \%$ & $57,5 \%$ & $42,5 \%$ & $79,2 \%$ & $20,8 \%$ \\
\hline Uso de peças sintéticas & $41,7 \%$ & $58,3 \%$ & $50,8 \%$ & $49,2 \%$ & $57,5 \%$ & $42,5 \%$ \\
\hline Laboratório nas monitorias & $71,7 \%$ & $28,3 \%$ & $73,3 \%$ & $26,7 \%$ & $88,3 \%$ & $11,7 \%$ \\
\hline
\end{tabular}

Anatomia I (anatomia básica dos sistemas do corpo humano); Anatomia II (anatomia do sistema musculoesquelético); Anatomia III (neuroanatomia). 
atuação da graduação em questão, permitindo que maiores links possam ser feitos pelos alunos quando virem as demais disciplinas".

Em outro trabalho ${ }^{17}$, neste contexto de sugestões para melhor aprendizado na disciplina de Anatomia Humana, os alunos do estudo sugeriram aumentar os intervalos entre as avaliações e o tempo necessário a sua realização, além de aplicar exames orais e fixar critérios para a execução das avaliações. Em outros trabalhos, mencionaram que a metodologia empregada deveria integrar os alunos de forma dinâmica, independentemente do método do professor, além de citarem que a importância do ensino da anatomia palpatória no curso de Fisioterapia deveria ser enfatizada. Além do conhecimento clínico, o estudante de Fisioterapia necessita ter o conhecimento prático do toque ao examinar os pacientes, tendo, desta forma, uma Anatomia mais dinâmica e correlacionada com a profissão $^{14,3}$.

Já na quarta parte do questionário, referente à avaliação do docente, os discentes deveriam se manifestar quanto à satisfação ou insatisfação em alguns quesitos demonstrados na tabela 2. De modo geral, os dados sugerem uma pequena superioridade na taxa média de alunos satisfeitos (52,63\%) ao se analisarem conjuntamente as três Anatomias. No entanto, as notas dadas pelos alunos aos professores das Anatomias I, II e III, por parte da avaliação promovida pelo colegiado de Fisioterapia, corresponderam às médias de 9,4, 8,5 e 7,9, respec- tivamente. Foi observada ainda uma taxa média de satisfação de $85,27 \%$ para o domínio do conteúdo e de $85,57 \%$ para assiduidade. Por outro lado, dentre outros quesitos, foram observadas taxas de insatisfação média entre as três Anatomias de $77,23 \%$ para a avaliação que prioriza a memorização ao invés de raciocínio e de 63,07\% no quesito qualidade da avaliação.

Buscou-se identificar algumas correlações pertinentes ao estudo, como: (a) rendimento final e afinidade com a matéria de Anatomia Humana, observando-se correlações fracas positivas em todas as disciplinas (Anatomia I $r=0,08$; Anatomia II $\mathrm{r}=0,14$; Anatomia III $\mathrm{r}=0,17$ ); (b) rendimento final e dificuldade de aprendizagem, apresentando correlações fracas negativas (Anatomia I $\mathrm{r}=-0,25$; Anatomia II $\mathrm{r}=-0,26$; Anatomia III $\mathrm{r}=-0,09$ ); (c) rendimento final e conteúdo ministrado, observando-se correlações fracas positivas em Anatomia I ( $\mathrm{r}=$ $0,07)$ e Anatomia II $(\mathrm{r}=0,11)$ e fracas negativas em Anatomia III ( $\mathrm{r}=-0,07)$; $(\mathrm{d})$ rendimento final e método de avaliação do conteúdo, observando-se correlações fracas positivas em Anatomia I $(r=0,21)$ e Anatomia II $(r=0,36)$ e fracas negativas em Anatomia III $(\mathrm{r}=-0,17)$; (e) rendimento final e didática, notando-se correlações fracas positivas em Anatomia I $(r=0,23)$ e Anatomia II $(r=0,29)$ e fracas negativas em Anatomia III ( $r$ $=-0,15)$; (f) rendimento final e qualidade dos instrumentos de avaliação, notando-se correlações fracas positivas em Anatomia I $(r=0,16)$ e Anatomia II $(r=0,30)$ e fracas negativas em Anatomia III $(\mathrm{r}=-0,21)$; $(\mathrm{g})$ rendimento final dos alunos re-

TABELA 2

Satisfação dos discentes do curso de bacharelado em Fisioterapia quanto à avaliação do docente ministrante da disciplina Anatomia Humana

\begin{tabular}{|c|c|c|c|c|c|c|}
\hline & \multicolumn{2}{|c|}{ Anatomia I } & \multicolumn{2}{|c|}{ Anatomia II } & \multicolumn{2}{|c|}{ Anatomia III } \\
\hline & Insatisfeito & Satisfeito & Insatisfeito & Satisfeito & Insatisfeito & Satisfeito \\
\hline Didática & $40,8 \%$ & $59,2 \%$ & $53,3 \%$ & $46,7 \%$ & $58,3 \%$ & $41,7 \%$ \\
\hline Organização & $17,5 \%$ & $82,5 \%$ & $20,8 \%$ & $79,2 \%$ & $54,2 \%$ & $45,8 \%$ \\
\hline Assiduidade & $3,3 \%$ & $96,7 \%$ & $5,0 \%$ & $95,0 \%$ & $35,0 \%$ & $65,0 \%$ \\
\hline Qualidade da avaliação & $57,5 \%$ & $42,5 \%$ & $60,0 \%$ & $40,0 \%$ & $71,7 \%$ & $28,3 \%$ \\
\hline Domínio de conteúdo & $5,8 \%$ & $94,2 \%$ & $9,2 \%$ & $90,8 \%$ & $29,2 \%$ & $70,8 \%$ \\
\hline Discussão da avaliação & $51,7 \%$ & $48,3 \%$ & $53,3 \%$ & $46,7 \%$ & $60,0 \%$ & $40,0 \%$ \\
\hline $\begin{array}{l}\text { Técnicas adequadas ao } \\
\text { conteúdo }\end{array}$ & $47,5 \%$ & $52,5 \%$ & $62,5 \%$ & $37,5 \%$ & $67,5 \%$ & $32,5 \%$ \\
\hline $\begin{array}{l}\text { Avaliação: raciocínio } \\
\text { ou memorização }\end{array}$ & $76,7 \%$ & $23,3 \%$ & $80,0 \%$ & $20,0 \%$ & $75,0 \%$ & $25,0 \%$ \\
\hline $\begin{array}{l}\text { Conhecimento } \\
\text { anatômico e } \\
\text { Fisioterapia }\end{array}$ & $63,3 \%$ & $36,7 \%$ & $62,5 \%$ & $37,5 \%$ & $57,5 \%$ & $42,5 \%$ \\
\hline
\end{tabular}

Anatomia I (anatomia básica dos sistemas do corpo humano); Anatomia II (anatomia do sistema musculoesquelético); Anatomia III (neuroanatomia). 
provados e o grau de dificuldade desses alunos, observando-se correlações fracas negativas nas três disciplinas (Anatomia I $r=-0,30$; Anatomia II $r=-0,20$; Anatomia III $r=-0,10$ ); (h) rendimento final dos alunos reprovados e a afinidade pela disciplina, observando-se correlação fraca positiva em Anatomia I $(r=0,08)$ e correlações fracas negativas em Anatomia II ( $r$ $=-0,19)$ e Anatomia III $(r=-0,10)$. Portanto, não se percebe nenhuma correlação significativa entre os parâmetros acima relacionados. A baixa correlação e a fraca correlação negativa observadas sugerem que, diante das questões levantadas neste questionário, existe pouca robustez nos dados fornecidos pelos sujeitos deste estudo.

Após estes achados, pode-se inferir que o processo de ensino-aprendizado é bastante complexo, sendo compartilhado tanto pelo sujeito que ensina (docente), com seus conhecimentos e metodologia de como transpor o material de estudo, quanto pelo sujeito que aprende (discente), que retém o conhecimento passado e o transpõe no decorrer de sua vida acadêmica e posteriormente profissional. Espera-se que esse futuro profissional, formado não só nesta instituição, mas em todas as outras instituições de ensino, possa reconhecer o devido valor dessa matéria enquanto discente e se empenhar em aprendê-la e entender a aplicação de tais conhecimentos, para o benefício dos pacientes.

\section{CONCLUSÃO}

Com base nos resultados obtidos da amostra estudada, principalmente nos quesitos notas e aprovação, pode-se avaliar que houve aproveitamento teórico-prático satisfatório por parte dos discentes de Fisioterapia na disciplina Anatomia Humana. Todavia, tornou-se evidente a existência de descontentamento do alunato em relação à matéria, o que demonstra uma série de dificuldades em relação às disciplinas, ressaltando-se um alto grau de insatisfação em alguns quesitos, como a estrutura de ensino da disciplina.

Este estudo forneceu alguns subsídios para o aperfeiçoamento das disciplinas Anatomia Humana I, II e III no curso de bacharelado em Fisioterapia, apontando aspectos que podem ser melhorados na opinião dos discentes, que expressaram suas dificuldades e sugestões acerca dessas disciplinas básicas do curso.

\section{REFERÊNCIAS}

1. Gray H. Anatomia. Rio de Janeiro: Guanabara-Koogan; 1995.

2. Tavano RB, Oliveira MC. Surgimento e desenvolvimento da ciência anatômica. Anuário da Produção Acadêmica Docente 2009;2(3).
3. Souza RB, Carvalho RM, Capelli AMG, Ribeiro MC, Vandresen Filho S, Simionato C, et al. Eficacia del entrenamiento teórico-práctico en anatomía palpatoria empleada a los estudiantes de la carrera de fisioterapía. Int. J. Morphol 2004; 22(3)195-200.

4. Damasceno SAN, Coria-Sabini MA. Ensinar e aprender: saberes e práticas de professores de anatomia humana. Revista Psicopedagogia 2003;20(23).

5. Neves MVS. Uma nova proposta no ensino de Anatomia Humana: desafios e novas perspectivas. Volta Redonda; 2010. Mestrado [Dissertação] — Centro Universitário de Volta Redonda.

6. Montes MAA, Souza CTV. Inovações no processo ensino-aprendizagem no laboratório de anatomia humana: estratégias facilitadoras para a aprendizagem significativa. $5^{\circ}$ Encontro nacional de pesquisa em educação e ciências; 2005; Bauru, São Paulo, Brasil.

7. Costa GBF, Lins CCSA. O Cadáver no ensino da anatomia humana: uma visão metodologia e bioética. Rev Bras Educ Méd 2012; 36(3):369-373.

8. Tavano PT. Onde a morte se compraz em auxiliar a vida: A trajetória da disciplina de Anatomia Humana no currículo médico da primeira Faculdade oficial de Medicina de São Paulo - o período de Renato Locchi (1937-1955). São Paulo; 2011. Mestrado [Dissertação] — Universidade de São Paulo.

9. Puhl FW, Araújo MCP. Contribuições das atividades de monitoria no componente curricular de anatomia humana para a formação docente. $5^{\circ}$ Encontro regional sul de ensino de biologia, 4ํㅗำ Simsio Latino Americano e Caribenho de educação em ciências do International Council of Associations for Science Education; 2011 setembro. 18-21; Rio Grande do Sul, Brasil.

10. Montes MAA, Souza CTV. Estratégia de ensino-aprendizagem de anatomia humana para acadêmicos de medicina. São Paulo; 2009. Doutorado [Tese] — Instituto Oswaldo Cruz, Ensino em Biociências e Saúde.

11. Siqueira Neto EGB, Ferreira JR. O ensino da anatomia humana no curso de medicina da Universidade Federal de Goiás - Avaliação e Perspectivas. Arq. Ciênc. Saúde Unipar 2001; 5(1):41-50.

12. Abreu OL. Contribuição ao ensino de anatomia na formação do enfermeiro - um estudo direcionado a pratica de enfermagem em saúde pública na consulta pré-natal. Rio de Janeiro; 1991. Mestrado [Dissertação] — Escola de Enfermagem Anna Nery.

13. Castro SV. Anatomia Fundamental. Rio de Janeiro: McGraw-Hill; 1979. 
14. Piazza BL, Reppoldo Filho AR. O ensino da anatomia humana nos cursos de educação física da região metropolitana de Porto Alegre. Ciências em Movimento 2011 /2; 18(26).

15. Fornaziero CC, Gil CRR. Novas tecnologias aplicadas ao ensino da anatomia humana. Rev Bras Educ Méd 2003;27(2).

16. Vavruk JW. A importância do estudo da anatomia humana para o estudante da área de saúde. O Anatomista 2012; 3(2).

17. Barrovecchio Jc, Perez B, Bella de Paz L. Sugerencias acerca del proceso de enseñanza-aprendizaje en Anatomía Humana. Rev. Chil. Anat 1998;16(2):219-224.

\section{CONTRIBUIÇÃO DOS AUTORES}

Os autores participaram de forma suficiente na concepção e desenho deste estudo. Rodrigo Moreira Arruda aplicou os questionários e escreveu o artigo. Cintia Regina Andrade Sousa analisou e interpretou os dados, bem como fez a revisão final deste texto.

\section{CONFLITO DE INTERESSES}

Não existem conflitos de interesses de qualquer um dos autores.

\section{ENDEREÇO PARA CORRESPONDÊNCIA}

Rodrigo Moreira Arruda

Praça Sá Barreto, Número 110

Centro - Vitória da Conquista

CEP: 45000-620 - BA

E-mail: rodrigo.m.a.fisio@gmail.com 